\title{
Simulation of Sudden Water Pollution Accidents of Hun River Based on SMS
}

\author{
Bo Meng ${ }^{1,2}$, Nan Li ${ }^{1}$, Dongping Fang ${ }^{1}$ \\ ${ }^{1}$ Institute for Future Cites and Infrastructure, Tsinghua University, Beijing 100084, China \\ ${ }^{2}$ College of Land and Environment, Shenyang Agricultural University, Shenyang 110866, China
}

\begin{abstract}
This paper, based on the geographic information and hydrological data, taking Shenyang section of Hun River Basin as study area, has introduced SMS software to set up the regional digital model. During the establishment of digital triangle mesh, geographic data is created, transformed, adjusted and revised. The two-dimensional hydrodynamic module RMA2 and water quality module RMA4 in the software are both used to build the hydrodynamic model and water quality model. On this basis, this papersimulates migration and transformation of pollutants in the river when pesticide 2,4-D polluted into the river. Besides, this article creates images to display the special and temporal changes in pesticide concentration visually. It also shows the process of contaminants flow through the city of Shenyang. According to the simulation results, sudden water pollution accident response strategies are explored, which can provide scientific technology and practical support about the emergency warning management system for the governmental departments.
\end{abstract}

Keywords: Hun River; Sudden water pollution; Simulation; SMS

\section{Introduction}

Water is an essential resource for the survival and industrial development of human society. The quality of water is not only related to the development and progress of a region, but also crucial to social stability. In recent decades, with the rapid development of China's economy and the growth of human activities, the conflict between people and the environment is becoming more and more fierce. The environmental pollution problems, especially the water environment problems, are becoming increasingly severe. As the main gathering environment of pollutants, water environment is more fragile than air and soil, which means it is easier to break the water ecological balance due to external interference. As a natural resource closely related to human beings, water resource often causes serious consequences because of non-adequate protection. Once a part of water body is polluted, it will also become a potential pollution source, lead to a vicious circle of pollution. Therefore, it is essentially necessary to strengthen monitoring and treatment of water environment. In all the water pollution types, accidental water pollution leads the most serious consequence[1]-[3].

Nowadays, the study of sudden water pollution accidents usually contains three aspects: mathematical model establishment, risk analysis and prediction, emergency warning management[4]. Once a hydrodynamic - water quality model of a water area is established, computer simulation can be used to reproduce the water quality of the area repeatedly. The hydrodynamic - water quality model can take many factors such as time, space and pollutants as control conditions to simulate the space-time diffusion with different locations, different time and various intruding contaminative substances[5]- [6].By using this model and the 
ground monitoring network, we can not only provide a scientific decision-making plan before water pollution events, but also it is possible to make a timely prediction after the events, then provide a reliable reference for the relevant governmental departments[7].

\section{Materials and methods}

\subsection{An overview of the research area}

The Hun River Basin (Fig 1) is located in the central Liaoning province of China, with geographical range $122.13^{\circ} \mathrm{E}-125.21^{\circ} \mathrm{E}$, $40.71^{\circ} \mathrm{N}-42.17^{\circ} \mathrm{N}$. The basin area is 28260 cubic kilometers, the total length is 495 kilometers, and the average annual precipitation is $742 \mathrm{~mm}$. The upstream of Hun River Basin mainly comprises Mountain Hilly Terrain, occupies $65 \%$ of the total area of the basin. The natural vegetation type is broadleaved deciduous forest, and the protection is relatively complete, with the vegetation coverage rate over $50 \%$. The middle and downstream of the basin are the urban plain, which occupies $35 \%$ of the area of the basin. This urban plain is an important economic core area with high degree of land development[8].
2. 2 The establishment of hydrodynamic system of study area

\subsubsection{RMA2 model and equations}

RMA2 is a two-dimensional finite element hydrodynamic numerical model developed by the Norton, King and Orlob of the US Army Corps of Engineers Waterway Experimental Station(USACE-WES), which was published in 1973. The code of the model has been modified and optimized several times by the American Water Resources Management Association (RMA) and the USACE-WES. At present, it has become one of the main hydrodynamic models in the industry[9]-[11].

2.2.2 Data acquisition and processing in prophase

In order to create available input data for SMS software, we need to obtain the channel information of Hun River in Shenyang section. However, there is no digital data of Hun river can be used directly, we need to create it firstly. In this study, the digital model of the Shenyang section of the Hun River is established by the steps of manually delimiting the area of the river surface by the satellite map; intercepting the altitude of river; processing altitude scatter points for the use of SMS software.

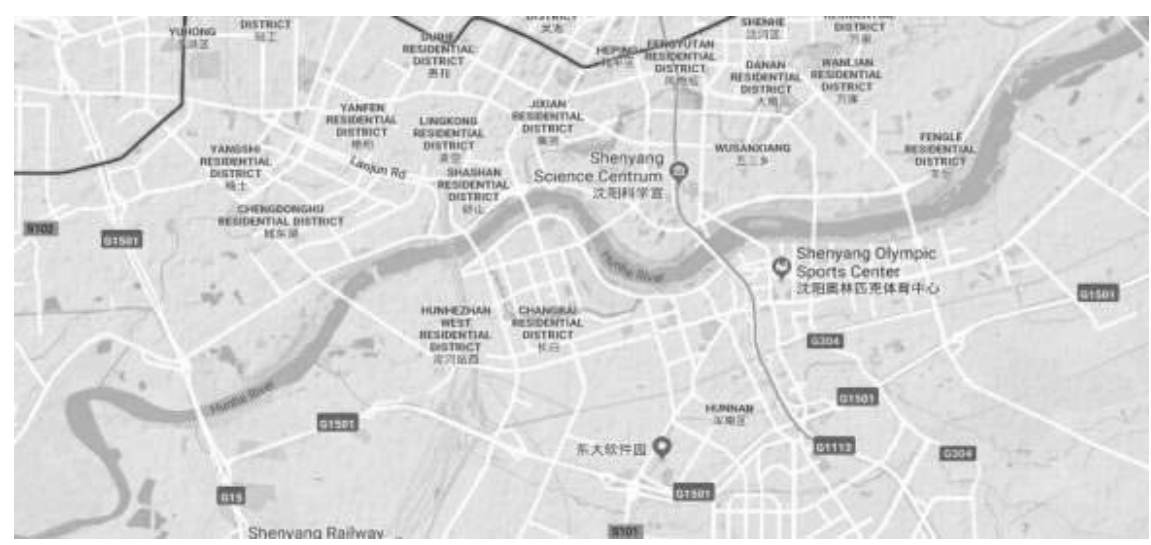

Fig1 The Hun River Basin 


\section{Material processing}

Because the river surface image need to be added to the satellite map, we need to stitch the pictures into a single picture, and then compress the picture size to be available. Finally, the river surface is created in ArcGIS. Open a new Shapefile, create polygon along the river bank of satellite map, so as to cover the Hun River study area, starting from Gaokan Bridge of the Forth Ring Road in the east, ending at the Hun River Gate Bridge of Metropolitan Beltway Highway in the west. The total length is about 37 kilometers, and the area is about 17 square kilometers.

\section{Data import and mesh generation}

The altitude file with xyz format is loaded with the Import Wizard module in SMS software, and imported in scatter point format, Through the input of geographic information, the geographic data is input into the module, and a series of data processing processes, including boundary processing and grid partition, are established.

\section{Model validation and map fitting}

After establishing the mesh model, we need to check whether the model matches the actual river situation. We used coefficient amplification instead of projection transformation to coordinates during importing, so we can conveniently apply inside software verification or outside software verification. If the outside software verification is applied, we can reduce the reverse coefficient, so that the coordinates are returned to the original geographic projection system WGS1984, and the numerical value is the latitude and longitude, which is derived as the $\mathrm{kmz}$ grating format. Open in Google Earth, it basically fits with the satellite map.

If we use inside software verification, we need to import the satellite map into the Map module of SMS software with the same coefficient amplification. The basic operation is similar to the ArcGIS software, which means 3 points are needed for coordinate registration.
Here we select three vertex angles and input the corresponding enlarged coordinates, and then superimpose the satellite map. As shown in Fig 5, after checking, we find that the river channel was completely covered, there is no over range area nor non-calculation area. The model validation is completed.

\subsubsection{Hydrodynamic operation based on} RAM2

First, we need to set up the RMA2 module, configure the inflow boundary and the outflow boundary. According to the results of Liaoning water conservancy general investigation in 2011, the inflow boundary is set to stable traffic volume $8000 \mathrm{~m} 3 / \mathrm{s}$, based on typical traffic flow of Hun River lockage statistics. The outflow boundary is configured as water level $25 \mathrm{~m}$ according to hydrological information. In the model control, according to the software manual, the cold boot water level is set up as $30 \mathrm{~m}$, the water temperature is $15^{\circ} \mathrm{C}$, the eddy viscosity coefficient is set up as recommended (1000 for both $\mathrm{x}$ and $\mathrm{y}$ direction), the Manning roughness coefficient is set up as 0.25 (ref. the existing study on Hun River in Fushun), the iteration number is set up as 10000 times, the model convergence condition is 0.001 , the time step is $0.5 \mathrm{~h}$, simulate $24 \mathrm{~h}$, and 48 times calculation in total. At the same time, the test of dry-wet conditions is operated to ensure the accuracy of the calculation to avoid the low water level and the emergence ofisolated island. Finally, we can exclude potential computing problems through RMA2 model checking, and confirm that RMA2 operation can be done without mistake.

\subsubsection{Model verification}

In order to ensure that the model is conformed to the actual situation and guarantee the accuracy of water quality model calculation, we must verify the hydrodynamic model. The hydrological data of Shenyang section of Hun River is applied for the model verification. The name of the hydrologic station is Hun River Shenyang section (III), the catchment area is 
7919 square kilometers, the coordinate is $123^{\circ}$ $23^{\prime} 19.9^{\prime \prime} \mathrm{E}, 41^{\circ} 45^{\prime} 28.3^{\prime \prime} \mathrm{N}$, the observation item is 111001 . The input data is the mean value of hydrological station flow from January to December in 2011. The output data is the corresponding hydrologic station measured flow velocity and the calculated flow velocity by RMA2 model [32]. By comparison, it is found that the error is less than $10 \%$, which illustrates that it is well fitted with hydrodynamic model. The simulation of water quality can be carried out because the simulation requirements are met completely.

4 Case simulation of pollutant diffusion based on RMA4

We apply a hypothetical case: A transport vehicle loaded pesticide 2,4-D has traffic accidents, all pesticide leaks into the Hun River. The diffusion of pesticide 2,4-D in water is simulated by the hydrodynamic system of Hun River Basin established before and the water quality module RMA4.

Before RMA4 water quality calculation, hydrodynamic modeling is first needed. Usually the calculation unit is the same as the RMA2 hydrodynamic calculation. The modeling is mainly composed of two-dimension units, which assumes that all elements of water quality are evenly distributed along the depth of water. The pollutants in RMA4 are realized by adding pollution to a certain unit on the boundary and increasing pollution inflow; the pollutants brought in with the flow are realized by entering the pollutant concentration to the boundary of the pollution inflow.

4.1 Establishment and calculation of water quality model

The RMA4 module uses RMA2 calculation results, and the pollutant inflow boundary is set in the same way, which means select the same area as the RMA2 inflow boundary. In order to simulate the pesticide entering accident, the pollutant concentration is not a fixed value. The change of concentration with time as shown inFig2.

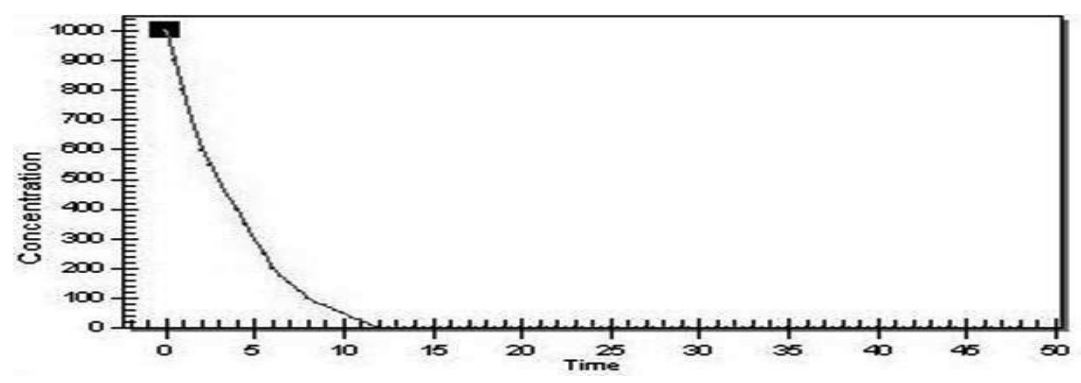

Fig 2 Inflow concentration setting

\subsection{Visual output of simulation results}

After the simulation, the observation points are set up at the $1 \mathrm{~km}, 2 \mathrm{~km}, 5 \mathrm{~km}, 10 \mathrm{~km}, 20 \mathrm{~km}$,
$30 \mathrm{~km}$ and outflow boundary from the accident place. The concentration of each observation point varies with time as shown in Fig3. 


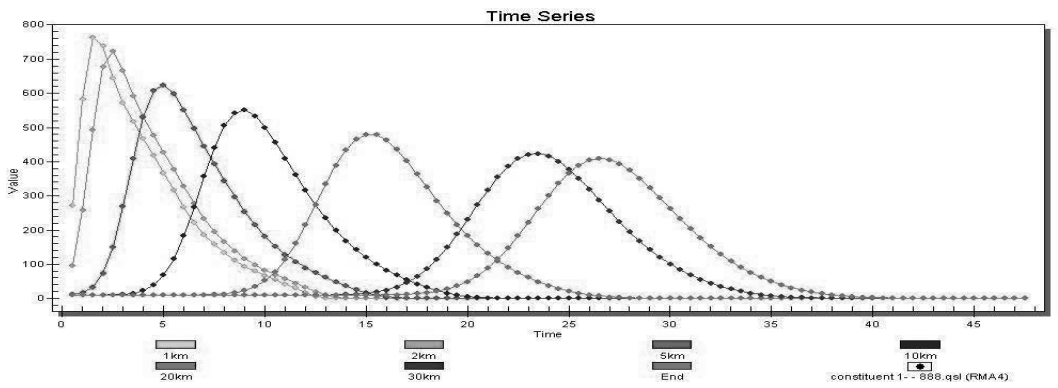

Fig 3 The various concentration with time of different observation points

In addition to generating distance plan for pollution accidents. Concentration is -concentration figures, the software can also generally shown by color map, red is high create dynamic video files, which show more concentration, while blue is low concentration. 1 clearly the spatial and temporal migration of pollutant dispersion. It is also possible to analyze hours, 5 hours, 10 hours and 20 hours after accident, the pollutant concentrations of rivers the surrounding facilities more accurately, in change as follows (Fig4-Fig7). order to make disaster analysis and emergency

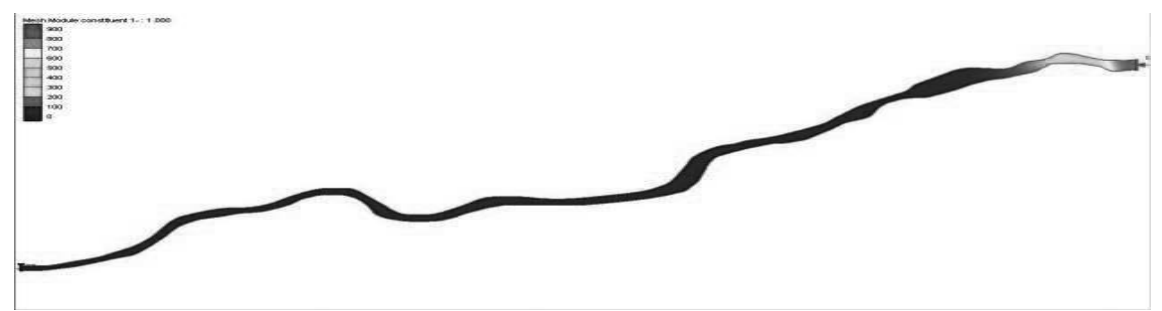

Fig 4 Concentration distribution 1 hour after accident;

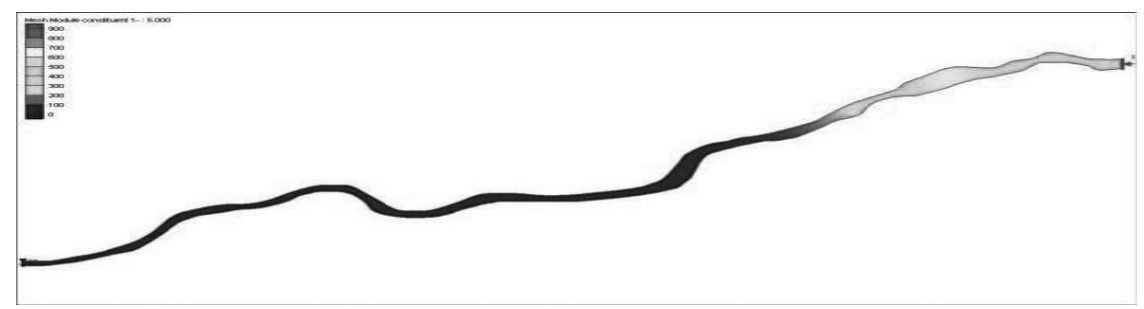

Fig5 Concentration distribution 5 hours after accident

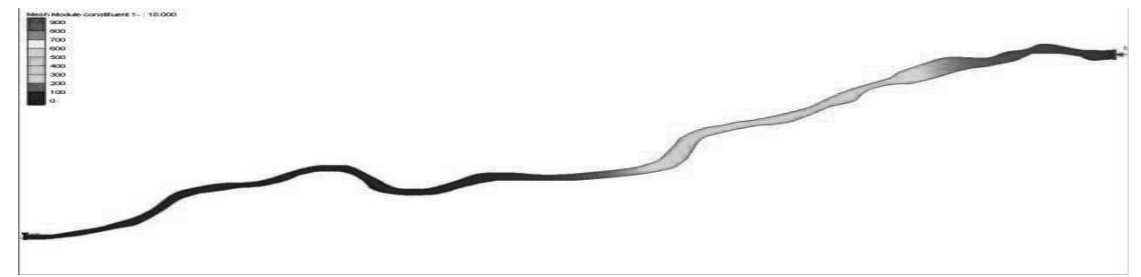

Fig 6 Concentration distribution 10 hours after accident; 


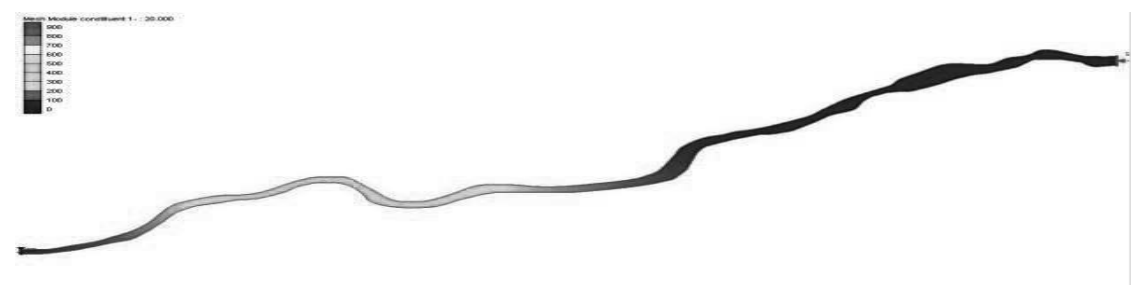

Fig 7Concentration distribution 20 hours after accident

When the peak of the pollutant flows through the Shenyang hydrological station of the Hun River, the accident has happened 19.5 hours; when it flows out from the hydrological station, the accident has happened 23.5 hours. The peak of the concentration is 400 units. The analysis of the superimposed satellite image is shown in Fig8.

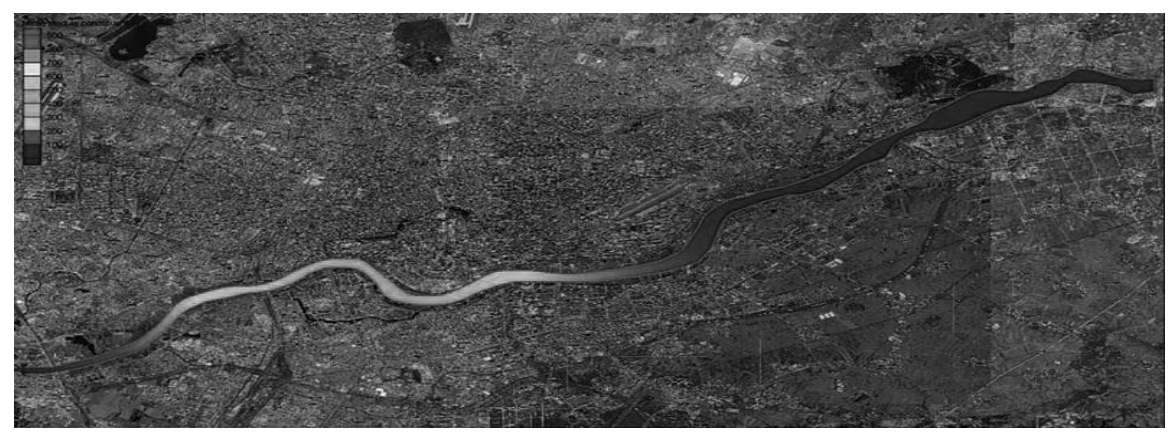

Fig 8 Superimposed satellite image analysis

\section{Conclusion and discussion}

\subsection{Simulation results analysis}

According to the results of the water quality simulation, we can see that after the sudden water pollution accident, the pesticide 2,4-D enters the river. When the pollutant is completely diffused, the length of the total pollution zone can reach 10 kilometers, but the area with the peak of the concentration of more than 600 units is only 5 kilometers away from the accident place. 10 hours after the accident happens, the peak concentration drops to half of the original value. 19.5 hours after the accident, pollutant peak concentration drops to 450 units, through the Shenyang monitoring station of Hun River. 32 hours after the accident, the pollutant completely flows out of the research area.

By contrasting the map, it illustrates that the pollutant inflow has the greatest impact on Shenyang Bird Island Nature Reserve. 30 minutes after the accident, pollutants arrive at Bird Island, the peak value of pollutant concentration reaches 800 units, which will cause huge damage to the ecological environment of Bird Island.

The agricultural water in the surrounding villages and towns will also be impacted. There are many villages and agricultural land in the southern bank of the Hun River, and the water intakes cannot be accurately identified, but the river pools, the low-lying land and the crop planting areas are obviously visible from the satellite map. There is a wide range of farmland within 15 kilometers from the accident place, which will be exposed with pollutants immediately after the accident. 14 hours after the accident, the peak concentration of pollutant will enter the hardened embankment of the city. So early warning of agricultural water pollution should be extremely necessary.

According to the information from water 
supply factory, drinking water is taken from underground, both for the urban area of Shenyang City and the surrounding rural areas. Therefore, the sudden water pollution accident will not cause pollution to the drinking water immediately. However, it is still necessary to closely monitor the water quality indicators of the intakes to prevent possible seepage pollution. The water quality early warning can be relieved 96 hours after the accident when the overall pollution is eliminated.

The pollutant diffusion is very sensitive to water flow after several times' simulations. When the flow rate is reduced from $8000 \mathrm{~m} 3 / \mathrm{s}$ to $7000 \mathrm{~m} 3 / \mathrm{s}$, the pollutant will pass more than 48 hours through the observation area. While when increasing the flow rate from $8000 \mathrm{~m} 3 / \mathrm{s}$ to 9000 $\mathrm{m} 3 / \mathrm{s}$, the pollutant can completely flow out only in 24 hours. Therefore, according to the requirement of pollutant controlling, we can adjust the flow rate of water gates and the upstream sluices, even adjust the flow rate of Dahuofang reservoir if necessary, in order to accelerate the diffusion of pollutants or to restrict the pollutant to a certain area for centralized disposal.

\subsection{Conclusion and research prospect}

This paper assumes that pesticide leakage is caused by traffic accidents during the transportation of pesticides; and simulates the sudden water pollution accident in Shenyang section of Hun River. Using the two-dimensional hydrodynamic module RMA2 and the two-dimensional water quality module RMA4 in the SMS surface water simulation software system, the sudden water pollution accident caused by the overturning of transportation vehicle with pesticide 2,4-D is simulated, and the area covers the Shenyang section of Hun River and Metropolitan Beltway Highway. With the successful establishment of hydrodynamic water quality model, the spatial and temporal migration and transformation of pesticide contaminants in the river are studied. It is proved that with the application of SMS software, the relevant water quality data can be obtained based on the existing hydrology, water quality and topographic data, and the data is quite reliable and practical. The simulation and operation of hydrodynamic - water quality model can effectively reduce the consumption of manpower and material resources, shorten the study time, and deal with sudden water pollution accidents more efficiently.

\section{Reference}

[1] Cao B Q, Jia H. Emergency treatment for sudden water pollution in central urban area of Nanyang City. Journal of Yangtze River Scientific Research Institute,2011(8): 72-76.

[2] Zhang B, Qin Y, Huang $M$, et al. SD-GIS-based temporal-spatial simulation of water quality in sudden water pollution accidents. Computers \& geosciences, 2011, 37(7): 874-882.

[3] Tang C, Yi Y, Yang Z, et al. Water pollution risk simulation and prediction in the main canal of the South-to-North Water Transfer Project. Journal of Hydrology, 2014, 519: 2111-2120.

[4] Zhang R, Zou H, Hong M, et al. Risk analysis of water resources crisis in the Lancang-Mekong river drainage basin under the background of climate change. Journal of Risk Analysis and Crisis Response, 2012, 2(3): 209-213.

[5] Ren X, Yu D, Ruan Y, et al. Modeling of Municipal Drainage and Urban Channel Flooding in Coastal City in the South of China, Journal of Non-linear Mathematical Physics.2005,1: 146-162.

[6] K.W.Chau, Y.W.Jiang. Simulation of transboundary Pollutant transport action in the Pearl River delta.Chemosphere.2003(9):1615-1621.

[7] Steven D et al. A coastal perspective on security. Journal of Hazardous Materials, 2003, 104:1-13. 
[8] Lung W S, Larson C E. Water quality modeling of Upper Mississippi River and Lake Pepin. Journal of Environmental Engineering,1995,121(10):691-699.

[9] Polichtchouk Y. Geo-information systems and regional environmental prediction. Safety Science, 1998:30-63.

[10] US Army, Engineer Research and Development Center Waterways Experiment Station Coastal and Hydraulics Laboratory, Users to Guide to RMA2 WES Version4.5.2001.

[11] He J C, Li J. Concept of early-warning and emergency response system for sudden water pollution accident. Water Resources and Hydropower Engineering, 2005, 10: 023. 